\title{
COMPACT ELECTRON LINACS FOR RADIATION TECHNOLOGY SYSTEMS
}

\author{
V. Belugin, A. Mischenko, V. Pirozhenko*, N. Rozanov, A. Zavadtsev, \\ BioSterile Technology, Inc., Fort Wayne, USA \\ A. Korolev, K. Simonov, Istok, Frjazino, Russia
}

\begin{abstract}
Compact, self-contained electron beam systems are used for sterilization of medical devices and materials, treatment of medical and infectious wastes, non-intrusive inspection of cargo containers, etc. These systems, having massive local radiation shielding, require compact accelerators. 5-MeV and 7-MeV electron linacs are presented in this paper. The accelerators use standing wave biperiodic structure fed by magnetron generator with $2.8 \mathrm{GHz}$ frequency. The accelerators do not use an external magnetic field for the beam focusing. Bunching, acceleration and focusing of the beam are performed by $\mathrm{RF}$ field of the accelerating structure.
\end{abstract}

\section{INTRODUCTION}

We have been witnessing an evergrowing demand for the electron linear accelerators lately. They should be reasonably compact and offer a high beam power possible. The accelerators are required for the selfshielded systems that are intended for the radiation sterilization and non-intrusive inspection of cargo containers. Systems of this type ensure the radiation protection of the personnel and can be installed in unprotected areas. They can also be designed as mobile systems.

The systems of the electron energy about $5 \mathrm{MeV}$ are intended for the radiation sterilization of medical devices and materials at manufacturing facilities of small or medium size. They can also be used in clinics to sterilize medical devices and to treat medical and infectious wastes. This energy is large enough to obtain good distribution of radiation dose in depth of most standard boxes with medical devices. Usually two-sided irradiation is used to get the good use of the beam power. In particular, irradiation of syringes that are packed in boxes with $300 \mathrm{~mm}$ height and $0.1-0.15 \mathrm{~g} / \mathrm{ccm}$ bulk density, provides for acceptable dose distribution uniformity.

$7 \mathrm{MeV}$ energy systems are intended for inspection of containers. This energy ensures a sufficiently high yield of the x-rays providing an effective depth of up to 350 $\mathrm{mm}$ steel penetration.

Design, specific features and operating characteristics of $5-\mathrm{MeV}$ and $7-\mathrm{MeV}$ accelerators are presented in this paper. Some design characteristics of the linacs had been reported earlier [1].

* E-mail: v_pirojenko@mail.ru

\section{SYSTEMS CHARACTERISTICS}

In a self-shielded sterilization system, compact electron linac and irradiation chamber are located inside the radiation shield. The shield consists of a set of cast iron blocks and a built-in transport mechanism for conveying the irradiated objects. The following major features are characteristic for the systems:

- A highly effective sterilization of medical devices;

- Little time sterilization (1-2 min.);

- Minimum effect on the device properties;

- Reliable radiation safety;

- Full ecology safety.

Two types of sterilization systems have been developed. The general characteristics of these systems are presented in table 1 .

Table 1. Characteristics of sterilization systems

\begin{tabular}{|l|c|c|}
\hline \multicolumn{1}{|c|}{ Type of system } & SB 5000 & BIOSIRIS \\
\hline Electron energy (MeV) & \multicolumn{2}{|c|}{5} \\
\hline Beam power $(\mathrm{kW})$ & \multicolumn{2}{|c|}{2} \\
\hline Port dimensions $(\mathrm{m})$ & $0.6 \times 0.4 \times 0.3$ & $0.4 \times 0.3 \times 0.3$ \\
\hline $\begin{array}{l}\text { Processing unit } \\
\text { dimensions }(\mathrm{m})\end{array}$ & $4.8 \times 1.6 \times 2.7$ & $2.2 \times 1.8 \times 2.6$ \\
\hline
\end{tabular}

BIOSIRIS system is intended for clinics. It contains the transport mechanism in the form of a rotary table with two ports for the irradiated objects. When one port is under the electron beam, the other one can be reloaded.

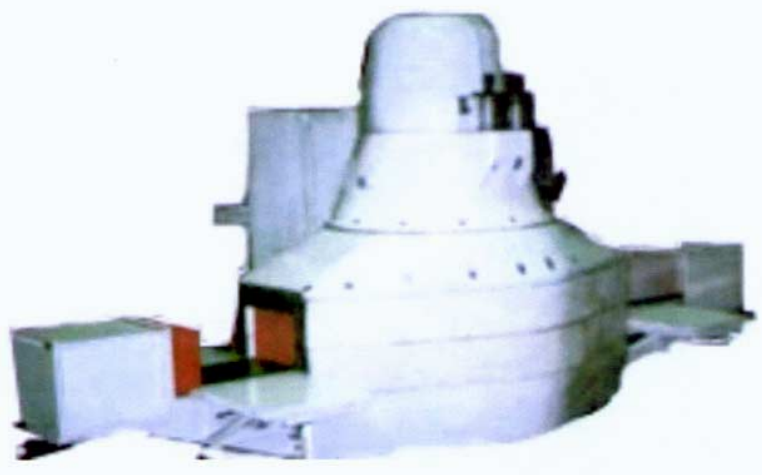

Fig. 1. SB 5000 system

SB 5000 system is intended for the manufacturing facilities. The system is shown in Fig. 1, the design of the processing unit - in Fig 2. The accelerator is located vertically. The electron beam goes down and is scanned 
transport mechanism contains 3 shielding blocks and 2 ports between them.

The accelerators operate continuously in each of the systems. The required shielding is assured at any position of the transport mechanism. To achieve a highly effective beam usage, the transport mechanism has two speeds: high speed when the object is being fed under the beam or low speed during the processing.

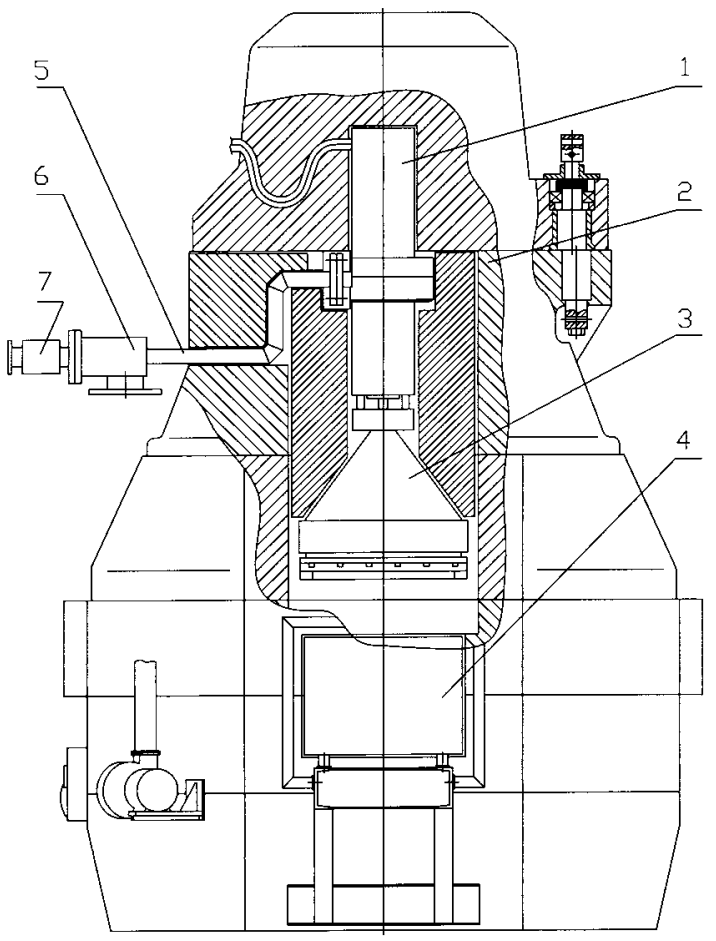

Fig. 2. SB 5000 processing unit

1 - accelerator, 2 - radiation shield, 3 - horn,

4 - irradiation chamber, 5 - waveguide,

6 - vacuum pumping section, 7 - ceramic window.

\section{LINEAR ACCELERATORS}

\subsection{Accelerator parameters and design}

The characteristic feature of the accelerators is that they do not use an external magnetic field for the beam focusing [2]. Bunching, acceleration and focusing of the beam are performed by RF field in the accelerating resonator.

The design of the accelerator is shown in Fig. 3a. The accelerator consists of the electron injector and accelerating resonator placed into the magnetic screen. The screen protects the accelerator from the outside magnetic fields, in particular, the residual magnetic field of the cast iron shielding blocks.
Major parameters of the accelerators used in the SB 5000 system and the INSIGHT inspection system are listed in table 2.

Table 2. Parameters of accelerators

\begin{tabular}{|l|c|c|}
\hline \multicolumn{1}{|c|}{ System } & SB 5000 & INSIGHT \\
\hline Maximum energy (MeV) & 5.2 & 7.3 \\
\hline $\begin{array}{l}\text { Pulse current of accelerated } \\
\text { beam (A) }\end{array}$ & 0.27 & 0.15 \\
\hline Beam pulse duration (mcs) & 5.5 & 11.5 \\
\hline Pulse repetition rate (pps) & 300 & 100 \\
\hline Length of linac (m) & 0.7 & 1.0 \\
\hline
\end{tabular}

The resonator is fed by the magnetron generator. The magnetron operates in "lock-on" regime. The waveguide system includes the ferrite circulator to ensure the matched load of the magnetron. The waveguide system includes also the vacuum window with ceramic insulator. Vacuum pumping of the accelerator is carried out through the waveguide.

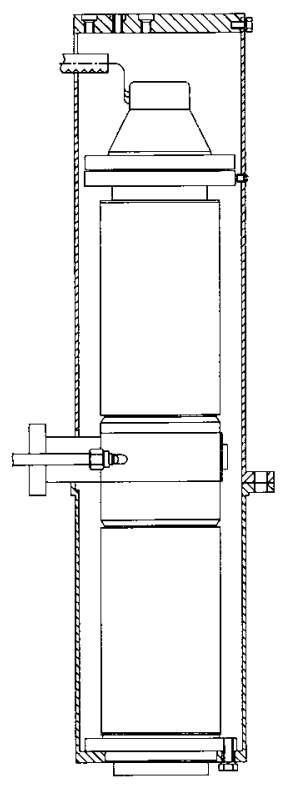

a)

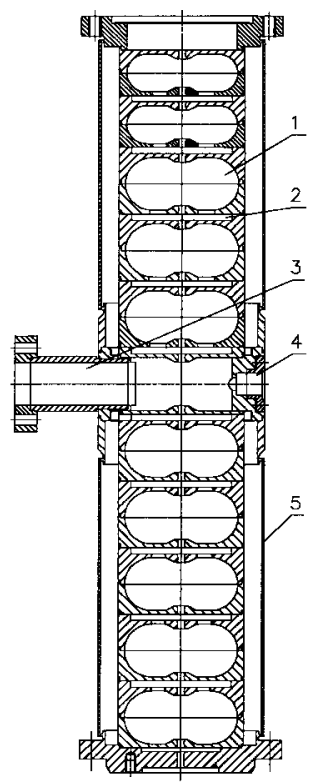

b)
Fig. 3. SB 5000 accelerator (a) and resonator (b). 1 - accelerating cell, 2 - coupling cell, 3 - waveguide, 4 - RF probe, 5 - water cooling casing.

\subsection{Electron injector}

The electron injector is located in the top part of the accelerator. It is connected directly to the resonator so that the first cell wall acts as the injector anode.

The injector contains the cone-shaped ceramic insulator, the cathode with filament, and the beam focusing electrode. Large diameter cathodes are used to obtain low current density and ensure high service life. The electron optics perform focusing beam so as current density at the resonator input is increased by a factor of 50 (table 3). 
Table 3. Parameters of electron injectors

\begin{tabular}{|l|c|c|}
\hline \multicolumn{1}{|c|}{ System } & SB 5000 & INSIGHT \\
\hline Electron energy (keV) & 40 & 40 \\
\hline Pulse beam current (A) & 1.5 & 0.8 \\
\hline Cathode diameter (mm) & 11.4 & 8 \\
\hline $\begin{array}{l}\text { Current density on the } \\
\left.\text { cathode (A/cm }{ }^{2}\right)\end{array}$ & 1.5 & 1.6 \\
\hline $\begin{array}{l}\text { Beam diameter at resonator } \\
\text { input (mm) }\end{array}$ & 1.5 & 2.0 \\
\hline
\end{tabular}

\subsection{Accelerating resonator}

The design of the accelerating resonator is shown in Fig. 3b. The resonator is the biperiodic structure operating in $\pi / 2$-mode. Major characteristics of the structure are specified in [3].

The accelerating cells have a common form of $\Omega$-shaped cross-section. The dimensions of the first two cells are less than those of the remaining cells.

The coupling cells are narrow cylindrical volumes. There are two coupling holes between accelerating and coupling cells. Additional holes are made in the resonator of the INSIGHT system to improve the vacuum conduction.

The resonator is cooled by water circulating inside the water casing. This ensures a good uniformity of the cell temperatures at high power operation.

$\mathrm{RF}$ power is supplied to the resonator by the waveguide connected to the middle cell through a rectangular-shaped window. The window dimensions have been adjusted to match the waveguide and resonator under full beam loading.

\begin{tabular}{|c|c|}
\hline System & \begin{tabular}{l|l} 
SB 5000 & INSIGHT \\
\end{tabular} \\
\hline Operating frequency $(\mathrm{MHz})$ & 2797 \\
\hline $\begin{array}{l}\text { Effective shunt impedance } \\
(\mathrm{MOhm} / \mathrm{m})\end{array}$ & 70 \\
\hline Coupling coefficient & $3 \%$ \\
\hline Number of accelerating cells & 17 \\
\hline
\end{tabular}

\subsection{Beam dynamics features}

The electron beam acceleration without special focusing means has been known for a long time [2]. Now the challenge is to achieve a high power beam in accelerators of this type. To optimize the acceleration process, a great number of calculations of the beam dynamics have been performed using "large particles" approach.

The major problem was to obtain an acceptable capture of the injected electrons into the acceleration process and perform the beam acceleration with a minimal loss of particles. The problem has been solved mainly by selection of dimensions of the first two cells and field levels in the cells.

The capture efficiency is about $20 \%$. The rest of the electrons fall on the walls. As they are capable of initiating a high-voltage breakdown, the field intensity in the first cells is a little reduced.

Of fundamental importance is the selection of phases of particles passing the resonator gaps. At the most part of the accelerator, the particles are located on the focusing slope of RF field. The values of the phases are modest in order to provide high acceleration rate, but the beam focusing is rather strong. On Fig. 4 are shown the radial profiles of the beam density at the accelerator output. The profiles have been calculated for two emittances of the injected beam. If the emittance is low, a thin core with high density takes place on the axis.

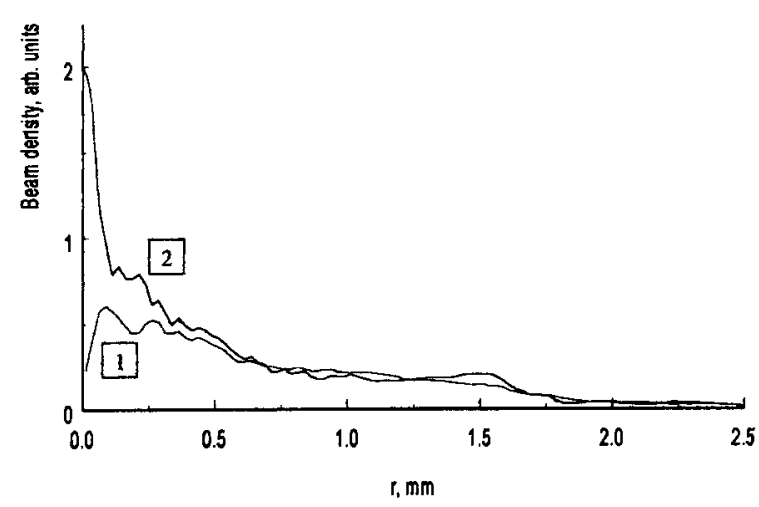

Fig. 4. Radial profiles of beam density for $45 \mathrm{~mm} \cdot \mathrm{mrad}(1)$ and $2 \mathrm{~mm} \cdot \mathrm{mrad}(2)$ emittances.

Selected character of the variation of the particles phases provides for high current of the beam, high acceleration rate, and good focusing of the beam with various emittances.

\section{CONCLUSIONS}

The electron linacs presented are reasonably compact and have sufficiently high beam power. They are selfshielded systems demonstrating good performance and reliable radiation safety. One of the systems has been in service for more than 5 years now.

\section{REFERENCES}

[1]. G.Batskikh et al. Proc. of 1999 Particle Acc. Conf., pp. 2564-2566.

[2]. K.Irie et al. Japanise Journ. Appl. Phys., 1973, v. 12, no 2, pp. 277-285.

[3]. A.Zavadtsev et al. Proc. of 1994 European Particle Acc. Conf., pp. 2564-2566. 